\title{
Risco de Sobrevivência de Micro e Pequenas Empresas Comerciais
}

\author{
Risk of Survival of Commercial Micro and Small Enterprises
}

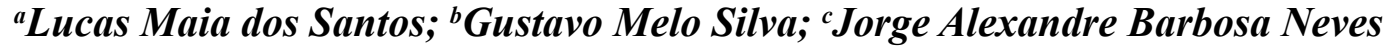 \\ ${ }^{a}$ Professor do Instituto Federal de Minas Gerais; Mestrado em andamento em Administração pela Universidade Federal de Minas Gerais, UFMG; \\ Viçosa, MG-Brasil; E-mail: lucas.maia@ifmg.edu.br. \\ ${ }^{b}$ Professor da Universidade Federal de São João del-Rei; Doutor em sociologia pela Universidade Federal de Minas Gerais, UFMG; \\ São João Del Rei, MG - Brasil; E-mail: gustavomelosilva@yahoo.com.br. \\ 'Professor da Universidade Federal de Minas Gerais, UFMG; Doutor em Sociologia pela University of Wisconsin-Madison/EUA, \\ Belo Horizonte, MG-Brasil; E-mail: jorgeaneves@uol.com.br
}

\section{Resumo}

O presente trabalho tem como objetivo investigar os fatores condicionantes do risco de sobrevivência das MPE comerciais da tecelagem tradicional de Resende Costa (MG). Os dados utilizados são referentes a uma pesquisa em 69 estabelecimentos comerciais, realizada no mês julho de 2009. Para operacionalizar o modelo de probabilidade foi utilizada a regressão logística em que a variável dependente se constituiu a partir de variáveis que poderiam reproduzir a organização e estrutura do comércio. O modelo obtido, baseado nos estudos de SEBRAE (2007), Lussie e Corman (2006) e Ercolin (2006), foi significativo para todos os testes de significância, com um poder preditivo de $94,2 \%$. As variáveis que representam a tendência de crescimento da comercialização, inovação dos produtos, tempo de atividade comercial, cursos e treinamentos definiram a função que possibilita a previsão de empreendimentos com maior probabilidade de sobrevivência no mercado. Desta maneira este estudo mostrou uma contribuição aos estudos sobre mortalidade das micro e pequenas empresas, sugerindo-se ampliação geográfica da amostra e das variáveis abordadas.

Palavras-chave: Micro e pequenas empresas; Risco de sobrevivência; Regressão logística.

\begin{abstract}
This study was carried out to investigate the conditioning factors of risk in survival of the weaving stores of Resende Costa (MG). The data come from a survey in 69 stores, held last July 2009. According to SEBRAE (2007), Lussie e Corman (2006) e Ercolin (2006), the model of logistic regression was used and the dependent variable was formed from variables that could reproduce the organization and structure of the enterprises. The model was significant for all tests of significance, with a predictive power of $94.2 \%$. The variables that represent the 'growing trend of trading', 'product innovation', 'time in the market', 'courses and training' defined the function that allows the prediction of enterprises most likely to survive in the marketplace. Thus this study showed a contribution to studies about mortality of micro and small enterprises, suggesting geographical expansion of both the sample and the variables.
\end{abstract}

Keywords: Micro and small enterprises; Risk in survival; Logistic regression. 


\section{INTRODUÇÃ̃o}

As micro e pequenas empresas formam um sistema produtivo no mundo inteiro que possibilita, na base de uma sociedade, a diluição da economia em milhares de empreendimentos. Destacando o caso brasileiro, as micro e pequenas empresas tem importância na geração de renda, na oferta de empregos e fortalecimento da economia. Por isso, este segmento tem um importante papel social e econômico, como pólo de criação e distribuição de riqueza, decisivo na geração de empregos e no desenvolvimento econômico.

De acordo com SEBRAE (2007), as MPE, em conjunto, responderam por 99,2\% do número total de empresas formais, por $57,2 \%$ do total de empregos e por $26 \%$ da massa salarial. Sem citar a grande massa de MPE que funcionam na informalidade, gerando renda, empregos e contribuindo para a redução da pobreza.

Uma pequena empresa, geralmente, é composta por uma equipe limitada em termos de quantidade de pessoas e, muitas vezes, também em termos de qualidade. Como a empresa não tem condições de contratar especialistas para suprir as necessidades, o próprio empresário torna-se polivalente, passando a atender problemas de produção, de comprar, de marketing de vendas e de recursos humanos (MARTENS, 2001).

De acordo Filardi (2006) e Ercolin (2007), muitos estudos concentram nos fatores que contribuem para o fracasso das empresas de médio e de grande porte. Poucos estudos nacionais e internacionais focaram esta problemática do ponto de vista da micro e da pequena empresa. Há, no entanto, um fato que compromete o maior crescimento do número absoluto de empresas e, por conseguinte da economia, que são os altos índices de mortalidade precoce de micro e pequenas empresas, ocasionados por diferentes elementos e condiçoes ligadas a estas unidades produtivas. Em cada 100 empresas abertas, segundo pesquisa do SEBRAE (2007), 31 não ultrapassam a barreira do primeiro ano de atividade.

De acordo com estudo recente sobre os fatores condicionantes e taxa de mortalidade das MPE, realizado pelo SEBRAE (2007), os próprios empresários apontaram as maiores dificuldades enfrentadas por eles, sendo que entre os fatores responsáveis pelo fechamento da empresa estão os seguintes problemas: carga tributária elevada e administração de capital de giro. A primeira razão apontada é externa à empresa, enquanto a segunda é inerente à gestão financeira do pequeno empresário e pode ser controlada internamente.

Disso, percebe-se que em razão das suas peculiaridades, as MPE enfrentam problemas bem específicos, em geral, diferentes ou em proporções diferentes quando comparados àqueles vivenciados pelas grandes empresas. Destaca-se que, às vezes, as MPE são atrativas do ponto de vista de rentabilidade, mas devido às deficiências na gestão financeira e operacional, operam de maneira ineficiente, o que as deixam suscetíveis a imprevistos comuns à natureza dos negócios. Isso instiga estudos voltados à análise das MPE, como proposto neste trabalho.

Visando contribuir com a análise da mortalidade das micro e pequenas empresas, o objeto de observação deste estudo são as MPE comerciais da tecelagem tradicional de Resende Costa (MG), que são uma fonte importante de trabalho e renda desta região. Neste contexto, este estudo quer resolver a seguinte questão: dentre algumas variáveis coletadas para este estudo, caracterizadoras de estrutura e gestão, existe pelo menos uma que poderia condicionar uma função de risco de sobrevivência nas MPE comerciais da tecelagem de Resende Costa (MG), com base no procedimento de regressão logística?

Portanto, o presente trabalho tem como objetivo geral investigar os fatores condicionantes 
do risco de sobrevivência das MPE comerciais de tecelagem tradicional de Resende Costa (MG). Para tanto, será utilizado como método a regressão logística para que se identifique e reconheça as variáveis que poderiam explicar o risco de sobrevivência, o que a nosso ver torna-se relevante para manutenção de empregos, geração de renda e redução dos níveis de pobreza. A categorização das variáveis relevantes que foram testadas neste trabalho por meio da ferramenta multivariada de regressão logística para identificação do risco de sobrevivência, fez-se uso de variáveis caracterizadoras das MPE conforme Bromley (1978), Amorim (2003), Bhowmik (2004), Custódio (2005), Chen (2007) e Sebrae (2007).

\section{A IMPORTÂNCIA DAS MICRO E PEQUENAS EMPRESAS}

Não há unanimidade sobre a delimitação do segmento das micro e pequenas empresas. Observa-se, na prática, uma variedade de critérios para a sua definição tanto por parte da legislação específica, como por parte de instituições financeiras oficiais e órgãos representativos do setor. A caracterização baseia-se no valor do faturamento, no número de pessoas ocupadas ou em ambos. A utilização de conceitos heterogêneos decorre do fato de que a finalidade e os objetivos das instituições que promovem seu enquadramento são distintos (ERCOLIN, 2007).

Conforme o disposto no artigo $3^{\circ}$ da Lei Geral das Micro e Pequenas Empresas, consideram-se microempresas aquelas que aufiram receita bruta igual ou inferior a $\mathrm{R} \$ 240.000,00$ em cada ano-calendário ou empresas de pequeno porte aquelas as que aufiram renda bruta anual superior a R\$240.000,00 e inferior a R\$2.400.000,00 (FILARDI, 2006; ERCOLIN 2007).

De acordo com Viapiana (2001), uma das dificuldades para estudar micro e pequenas empresas no Brasil aparece no momento de sua caracterização, visto que a adoção de diferentes parâmetros e critérios quantitativos e qualitativos por parte dos órgãos públicos oficiais, expande ou reduz a abrangência do conceito de micro e pequena empresa. No Brasil o ideal é considerar o valor do faturamento bruto e o número de funcionários, portanto, necessário recorrer a legislação, sendo que as esferas federais, estaduais e municipais possuem critérios diferentes. De acordo com Martens (2001), Filardi (2006) e Ercolin (2007), para ser classificada como micro empresa, a indústria deve possuir de 1 a 19 funcionários e a pequena empresa de 20 a 99 funcionários. No caso do comércio, a micro empresa deve possuir de 1 a 9 funcionários e a pequena empresa de 10 a 49 funcionários.

Lundvall (2001), Lemos (2002) e Campos (2003) citam alguns fatores que explicam com clareza o porquê de direcionar as políticas públicas de desenvolvimento a este segmento de empresas. Com relação à geração de empregos, estes autores explicam que a elasticidade emprego-produto é maior nos pequenos empreendimentos do que nas grandes firmas, ou seja, o aumento do emprego é mais que proporcional àquele decorrente na produção, sendo esta proporção mais acentuada nas micro e pequenas empresas. Portanto, as economias com presença marcante de micro e pequenas empresas sofrem aumento ainda maior dos postos de trabalho com o crescimento da produção, do que as economias com predomínio das grandes firmas expostas às mesmas situações de mercado.

Albagli e Brito (2002), Amorim (2003), Campos et al. (2003) afirmam que devido à flexibilidade, empresas menores podem trabalhar em um sistema mais dinâmico e moderno do aparelho produtivo, portanto, estas não são mais vistas como formas arcaicas e marginais de produção que apenas empregam grande volume de trabalhadores. A sua flexibilidade requer mão-de-obra em contínua formação, além de possibilitar um gerenciamento horizontal e 
descentralizado.

Para Lundvall (2001) e Lemos (2002), de maneira geral, as incertezas macroeconômicas da globalização da economia exigiram que se criasse um ambiente empresarial flexível o suficiente para enfrentar possíveis crises sistêmicas. Neste contexto, as pequenas firmas demonstram exímia competência em adaptar-se a ambientes diversos, com a rapidez necessária à manutenção da ordem econômica. Portanto, para estes autores esta realidade mostra a importância das pequenas firmas no combate à pobreza, afirmando que redistribuir renda requer redistribuição de ativos.

Lemos (2002) e Custódio (2005) apontam outro aspecto que confere importância às micro e pequenas empresas. Segundo eles, estas permitem a formação da classe empresarial nacional, por meio da absorção de tecnologia gerencial produzida em seu próprio ambiente. Além disso, viabilizam e facilitam a promoção da interiorização, o que fortalece as economias municipais à medida que utilizam matéria-prima e mão-de-obra local.

A partir da constatação da importância das micro e pequenas empresas, no contexto mundial e brasileiro, especialistas tem aplicado esforços no desenvolvimento de estudos, na tentativa de levantar o máximo de informações possíveis sobre este setor. Em recente estudo publicado pelo SEBRAE (2007) que mostra as características e fatores que influenciam a mortalidade das micro e pequenas empresas no Brasil, constatou-se que as empresas ativas, nascidas em 2005, possuíam em média 5 pessoas ocupadas por empresa, 94\% possuíam outros funcionários em atividade além dos sócios e 85\% possuíam empregados com carteira assinada.

Conforme o SEBRAE (2007), um grande número de micro e pequenas empresas morrem nos primeiros anos de existência apesar da redução do índice de mortalidade nos últimos anos. De acordo com o Gráfico 1 pode-se perceber que, no ano de 2003 esta taxa foi de $36 \%$, em 2004 de $31 \%$ e em 2005 de $22 \%$. Este mesmo estudo mostrou que entre as empresas que encerraram suas atividades em 2005, 14\% encerram no primeiro ano (2005), 52\% no segundo ano (2006) e $32 \%$ no terceiro ano (SEBRAE, 2007).

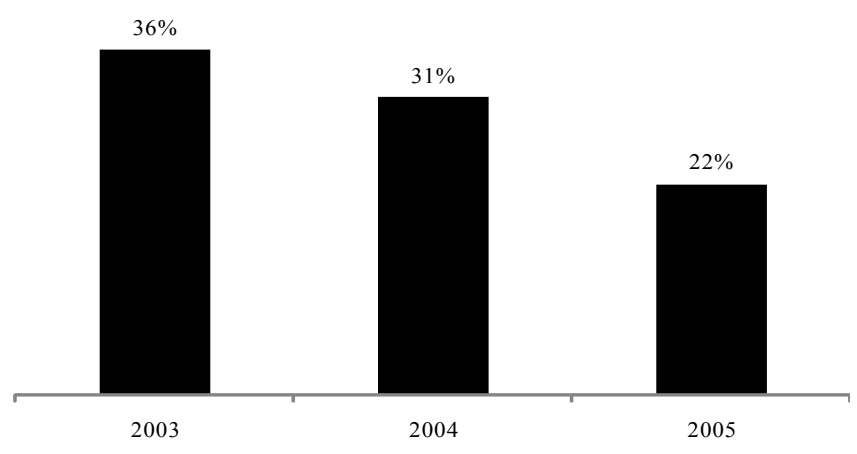

Gráfico 1 - Taxa de mortalidade de micro e pequenas empresas no Brasil

Fonte: SEBRAE (2007)

Entre os estudos internacionais que analisaram a mortalidade de micro e pequenas empresas cita-se Perry (2001) que afirma, com base em uma pesquisa que envolveu 152 empresas, que escrever um plano de negócios melhora o desempenho e reduz a probabilidade de morte das pequenas empresas, visto que mais de $50 \%$ das firmas pesquisadas que não fecharam, tinham escrito um antes de montar a empresa.

Cleverly (2002) indica os cinco fatores que, de acordo com seus estudos, contribuem para a mortalidade de empresas. São eles: a) elevado nível de complacência com os gastos na 
fase de abertura da empresas; b) nível de competência da diretoria e dos sócios varia muito, e muitos fazem parte da sociedade por causa do grau de parentesco, ou porque traz recursos financeiros, mas lhes falta experiência empresarial; c) em muitos casos, os novos empresários não tem recursos para abandonar seu atual emprego e se dedicar em tempo integral ao novo negócio; d) muitos não conhecem o mercado onde estão entrando e; e) muita informação e mudanças rápidas fazem com que os empresários não dêem foco ao que é prioridade.

Já os resultados de Mager (2002) demonstram que os principais fatores contribuintes para a mortalidade de pequenas empresas, conforme a seguir são: falta de planejamento estratégico de longo prazo, falta de conceitos de finanças, baixa qualidade da mão de obra, falta de processo e métodos internos de trabalho.

Riquelme e Watson (2002) concluíram que os principais fatores para a mortalidade de pequenas empresas são falta de experiência da equipe gerencial e da mão de obra, mercado com baixo potencial de crescimento e alta concorrência, produto sem diferencial competitivo, produto com baixa tecnologia e inovação.

Lussier e Corman (2006) concluiu que uma empresa de sucesso deveria ter, pelo menos, os lucros da média do setor. As empresas que falharam, por outro lado, são aquelas envolvidas em processos na corte ou ações envolvendo perdas a credores. Os fatores de sucesso analisados foram escolaridade, planejamento, número de funcionários, ambiente macroeconômico, sócios, idade do empreendedor, ajuda profissional, experiência prévia, marketing, dentre outros.

No Brasil, Filardi (2006) tomou as micro e pequenas empresas como base para responder se é possível identificar e caracterizar um conjunto de fatores contribuintes para a mortalidade precoce das micro e pequenas empresas e concluiu que fatores como gestão financeira, idade do proprietário, experiencia no negócio e setor de atuação são relevantes.

Ao contrário do que ocorre em outros países, principalmente nos Estados Unidos, o Brasil não possui muitos estudos com aplicação de metodologia de pesquisa científica sobre a mortalidade de pequenas empresas e não possui sistematização do estudo sobre este assunto, embora o SEBRAE tenha começado a estudá-lo.

O caso analisado neste trabalho, que se refere a MPE do mercado da tecelagem tradicional de Resende Costa (MG), compartilha uma realidade empresarial como as apontadas acima pelos autores que se referem ao contexto das MPE brasileiras. Pode-se afirmar que tais MPE possuem as mesmas dificuldades e peculiaridades, entretanto, este caso está relacionado a um pequeno município do interior do país que depende única e exclusivamente destes empreendimentos, já que sua economia se baseia na agricultura familiar e na prestação de serviços públicos.

\subsection{A tecelagem tradicional de Resende Costa (MG): as MPE locais de base artesanal}

A análise da cadeia de produção e de comercialização da tecelagem tradicional do município de Resende Costa (MG) pode ser caracterizada por pontos que estão estruturados nas variáveis históricas, sociais e culturais e no envolvimento produtivo de grande parte de sua população que envolvem, conforme Santos et al. (1998), diretamente 38\% dos moradores da sede do município. Além de estar aproximadamente há 20 anos enraizada nesta comunidade como uma atividade econômica e social de importância para a geração de trabalho e renda regional (Santos e Silva, 1997).

Os colonizadores trouxeram para este município, o conhecimento da tecelagem que, 
atualmente, movimenta sua economia, mas tanto o processo como os produtos vêm passando por modificações (ABREU, 2002). Sistemas produtivos que, inicialmente, eram desenvolvidos para auto-subsistência da propriedade rural são, atualmente, sofisticados na sua organização e nas relações de trabalho existentes. A organização do trabalho que era caracterizada por um artesão que dominava todo o processo de produção, atualmente é dividida e especializada por funções e produtos.

O desenvolvimento socioeconômico da rede organizacional desta indústria criativa pode ser compreendido a partir dos empreendimentos que se materializaram em novas combinações desenvolvidas pelos comerciantes locais, que atuam com sua racionalidade empreendedora de pequenos empresários. Como, por exemplo, no caso de Resende Costa em que a proximidade geográfica de pólos produtivos da indústria têxtil mineira de meados do século XX como São João del-Rei, Divinópolis e Juiz de Fora, possibilitou o aproveitamento do refugo das malharias como matéria-prima para sua produção de base artesanal (SANTOS e SILVA, 1997). Esta nova combinação da ferramenta tear de madeira com o refugo da indústria têxtil é fruto de tomadas de decisões, que foram realizadas por comerciantes artesãos com o intuito de viabilizar a produção em massa.

A divisão e especialização é um fato importante para compreender a realidade das mudanças que estão ocorrendo e viabilizando ganhos de produtividade via escala e diversificação de produtos nas organizações da indústria criativa local. Além de ser uma contradição que se coloca ao sistema de produção artesanal, já que o artesão clássico vem sendo substituído por classes de trabalhadores especializados em determinadas atividades do processo de produção ou até mesmo por produtos. Em pesquisa realizada por Santos et al. (1998) foram identificadas as seguintes funções na rede organizacional de produção artesanal de Resende Costa: picadores, tecelões, vendedores de artesanato e fornecedores de matérias-primas. Portanto, o que se observa é uma importante rede de MPE que formam uma cadeia de produção. A sobrevivência de cada elo é importante para sobrevivência de toda a economia municipal.

O que se observa nas relações de trabalho e produtivas na rede organizacional analisada é que há uma conciliação, mesmo que assimétrica, do interesse individual do empreendedor comerciante pela busca da maximização de seus lucros e sobrevivência, a partir de uma base comum de conhecimento produtivo que viabiliza a remuneração da força de trabalho local que é a detentora do saber artesanal. Tanto o trabalhador do sistema de produção como os empreendedores comerciantes são afetados em suas ações e atitudes por relações sociais, por exemplo, no recrutamento de trabalhadores ou na subcontratação de subunidades de certas famílias ou de indivíduos de certas localidades da zona rural do município que tem reconhecimento local de background artesanal.

A estruturação ou reestruturação da tecelagem tradicional em uma rede semi-autônoma hierarquizada de atores sociais, com papéis definidos no sistema produtivo ocorreu com a divisão e especialização do trabalho, que é um acervo comum do conhecimento produtivo comunitário deste município. Esta transformação de uma produção caseira e de subsistência para a principal atividade econômica do município e sua institucionalização é decorrente de fatos históricos como, por exemplo, a decadência da exploração do ouro. A busca pela sobrevivência dessas empresas em sua terra natal tornou natural o processo de transformação da produção artesanal em uma genuína manufatura têxtil que gera conflitos de poder econômico entre seus atores e que, por sua vez, demandam organizações que tem sua base estrutural na sociedade local. 


\section{MÉTODOS E PROCEDIMENTOS}

\subsection{Modelo de probabilidade de sobrevivência}

Para realização deste trabalho, uma amostra significativa de comerciantes foi entrevistada, intencionalmente, no comércio da tecelagem tradicional de Resende Costa. Os dados de origem das variáveis utilizadas neste estudo são referentes a uma pesquisa que visitou 76 estabelecimentos comerciais e aplicou um survey em 69 no mês de julho de 2009. Os entrevistados foram os proprietários dos estabelecimentos comerciais que escoam a produção da tecelagem tradicional domiciliar do município pesquisado. Para um parâmetro de comparação, conforme a Prefeitura Municipal de Resende Costa (2009), no dia 08 de julho de 2009 existiam 95 lojas de artigo de artesanato no município.

Para operacionalizar o modelo de risco de sobrevivência da atividade de comercialização de insumos e produtos acabados da tecelagem tradicional de Resende Costa, foi utilizado um modelo estatístico de probabilidade em que a variável dependente se constituiu a partir de variáveis que caracterizam a estrutura e a gestão das MPE, algumas utilizadas nos estudos de Perry (2001), Cleverly (2002), Mager (2002), Riquelme e Watson (2002), Lussier e Corman (2006) e Filardi (2006). As variáveis que foram obtidas nas entrevistas serão descritas posteriormente.

O objetivo da inclusão desse modelo como ferramenta empírica foi determinar as variáveis que estão mais associadas ao risco de sobrevivência destas micro e pequenas empresas, como proxy de sobrevivência. Assim, não constitui objetivo do estudo a identificação do efeito marginal destas sobre a variável dependente.

Como o problema remete à existência de variáveis categóricas, o teste de associação, bem como sua natureza, é realizado tomando como referência a distribuição de Qui-quadrado $\left(c^{2}\right)$, conforme procedimento destacado por Silver (2000). Nesse ponto, é oportuno destacar que, não existindo qualquer relação entre as variáveis independentes e a variável dependente,

o valor de $c^{2}$ será nulo. Caso contrário precisa-se apenas perceber se a diferença entre zero e o valor calculado, isto é, entre o valor observado e valor esperado, considerando as variáveis independentes do estudo, que são mais atribuíveis ao acaso ou erros de amostragem do que a dado nível de significância. Esse nível é normalmente oferecido como produto da operacionalização desse teste em maior parte dos softwares estatísticos, a exemplo do S-plus e o SPSS, muito utilizados em ciências sociais aplicadas.

De acordo com Gujarati (2000), nesses modelos, a variável dicotômica é uma variável dummy, que assume os valores 0 ou 1 , o que, na análise em questão, representa: 0 - com maior risco de sobrevivência e 1 - grupo com menor risco de sobrevivência. Neste estudo, a classificação no grupo com maior risco de sobrevivência não estabelece uma denominação literal. A classificação de algumas empresas neste grupo não supõe que estas empresas estão insolventes ou em decadência. Esta classificação apenas visa diferenciar entre um grupo que apresente mais ou menos características inerentes às micro empresas estabelecidas no mercado, baseados nos estudos sobre MPE.

Assim, no modelo Logit, define-se $P_{i}$ como a probabilidade de que a empresa apresente maior risco de sobrevivência. Essa probabilidade pode ser determinada por vários fatores (variáveis explicativas), representados por $X_{i}$, regredidos sobre a variável dependente $(\mathrm{Y})$. Dentre esses fatores, tem-se tempo de existência da empresa, faturamento bruto anual da empresa, número de empregos, entre outros. 
Uma vez que a probabilidade é uma variável que se encontra no intervalo [0,1], a relação entre essa probabilidade e determinado atributo pode ser representada. No caso do modelo Logit, essa relação é representada pela função densidade de probabilidade acumulada logística. Tem-se, assim: $P_{i}=f\left(X_{i} \mathrm{~b}\right)$ em que $f$ é uma função densidade probabilidade acumulada.

Nesse modelo, a variável dependente da regressão é o logaritmo das chances de um evento específico, no caso o risco de sobrevivência, conforme Equação 1:

$$
\mathrm{L}_{\mathrm{i}=} \mathrm{h}\left(\frac{P_{i}}{1-P_{i}}\right)=X_{i} \mathrm{~b}
$$

A equação 1 deve ser ajustada pelo método de Máxima Verossimilhança e não por Mínimos Quadrados Ordinários (MQO), como maior parte das regressões lineares. Para maiores detalhes sobre o modelo recomenda-se Gujarati (2000).

Por meio da obtenção dos coeficientes das variáveis explicativas, pode-se perceber o impacto dessas na variação do logaritmo da probabilidade da variável dependente, aqui compreendida como risco de sobrevivência.

\subsubsection{Operacionalização das variáveis}

Esperava-se, a priori, que fatores estruturais e organizacionais relacionados à importância da atividade para o empresário, caracterização da estrutura, administração, características da gestão e perfil gerencial das MPE pudessem, em menor ou maior escala, refletir o risco de sobrevivência dos estabelecimentos comerciais analisados. Devido à indisponibilidade de informações na coleta de dados da pesquisa, não foi possível incluir variáveis que representassem carga tributária e gestão de capital de giro.

Assim, com base nas referências dos estudiosos de MPE supracitados e nas características peculiares das MPE da amostra, esperava-se que o tempo de atividade comercial, importância da renda proveniente da atividade, treinamento, tendência de crescimento ou redução da produção, inovação nos produtos, número de empregados e receita mensal fossem significativos para explicar o fenômeno em questão, ou seja, se as informações sobre estas variáveis tem relação com fatores condicionantes de risco de sobrevivência das MPE.

A descrição da variável dependente, bem como as expectativas de relação das variáveis explicativas com o evento "risco de sobrevivência", serão mais bem exploradas a partir da exposição a seguir.

Risco de sobrevivência (Y): Representa uma proxy de sobrevivência, constatada por meio de informações obtidas na coleta de dados. Assim, a empresa que classificada no grupo com menor risco de sobrevivência tem como variável dependente $\mathrm{Y}=1$, caso contrário, classificada no grupo com maior risco de sobrevivência, $\mathrm{Y}=0$ à variável dependente. A construção da variável risco de sobrevivência $\mathrm{Y}$ foi constituída a partir da análise da composição de outras oito variáveis dicotômicas, denominadas $V_{i}(1 \leq i \leq 8)$, em que $V_{i}=0$ ou $V_{i}=1$. As variáveis $V_{i}$ assumiram os valores $V_{i}=1$ nas seguintes condições: empresas com mais de 5 anos de existência $\left(V_{1}\right)$, renda do comércio como principal fonte de composição da renda familiar $\left(V_{2}\right)$, participação em cursos e treinamentos $\left(V_{3}\right)$ filiação em associações ou cooperativas $\left(V_{4}\right)$, crescimento da produção $\left(V_{5)}\right.$, inovação nos produtos $\left(\mathrm{V}_{6}\right)$, mais de três empregados $\left(\mathrm{V}_{7}\right)$, renda média mais um 
desvio-padrão $\left(\mathrm{V}_{8}\right)$. A classificação, em que $\mathrm{Y}=1$, menor risco de sobrevivência para somatório de $V_{i} \geq 5$, e $\mathrm{Y}=0$ representou o maior risco de sobrevivência para somatório de $V_{i}<5$. Esse procedimento estabeleceu dois grupos de referência relativos para análise neste estudo: grupo com maior e menor risco de sobrevivência. Com esta classificação, aproximadamente, $39 \%$ da amostra foram classificadas com menor risco de sobrevivência $(\mathrm{Y}=1)$.

Tempo de atividade comercial (TAC): espera-se que empresas com mais tempo de atividade comercial sejam mais consolidadas, organizadas e com menor risco de sobrevivência. Também, espera-se que os problemas de capital de giro sejam menos frequentes por fatores como acesso facilitado a crédito, controles financeiros bem definidos, sólida participação de mercado, etc. Outro elemento de grande importância é a incorporação, com o passar do tempo, de conhecimentos acerca do negócio e do mercado onde atuam. Empresas com mais tempo de mercado ocupam lugar mais alto na curva de aprendizagem e, por conseguinte, são mais eficientes e possuem menores chances de fracasso, ou seja, menor risco de sobrevivência.

Status da renda (SR): trata-se de variável qualitativa em que se atribui 1=secundária, $2=$ sazonal e $3=$ principal para a importância da renda proveniente do comércio de artesanato. Assim, quanto maior o número (em uma escala de 1 a 3 ) maior é a importância desta atividade produtiva para a formação da renda familiar. Espera-se que nas empresas cujo comércio representa a renda principal, a dedicação do empresário seja maior. Portanto, atribui-se menor risco de sobrevivência de acordo com a maior importância do comércio na composição da renda familiar.

Curso ou treinamento (CT): variável qualitativa do tipo 1-sim ou 2-não. Sabe-se que novos conhecimentos são importantes para as melhorias de procedimentos e aplicações de novas técnicas de gestão ou técnicas produtivas que deixariam a atividade mais eficiente. Os comerciantes da tecelagem tradicional de Resende Costa (MG) podem encontrar em cursos, novas maneiras, procedimentos e mercado que possibilitam a melhoria contínua de suas atividades. Portanto, espera-se que as MPE que participaram de cursos e treinamento tenham menor risco de sobrevivência no mercado.

Filiação a associação (FA): variável qualitativa do tipo 1-sim ou 2-não. O associativismo e cooperativismo são outras maneiras de compartilhar o conhecimento das melhores práticas de produção e gestão e uma alternativa para ganhar em escala, seja no relacionamento com fornecedores ou clientes. Empresas que se associam ou cooperam estarão associadas a um menor risco sobrevivência.

Número de empregados (EP): esta variável é um importante indicador de porte das organizações. Por isso, espera-se que números maiores de empregados estejam associados à profissionalização da empresa. Empresas maiores têm maior movimentação financeira, sendo, por isso, beneficiadas por programas de relacionamento junto às instituições financeiras. Além disso, podem oferecer maior quantidade de garantias reais, o que significa, na prática, mais acesso ao crédito e melhores condições de pagamento. Além disso, as empresas maiores possuem estruturas mais adequadas, a exemplo de controles de estoques, políticas de crédito, entre outros elementos que permitem o uso mais eficiente de recursos. Neste sentido associa-se menor risco para mais empregados na empresa.

Faturamento bruto mensal (FBM): é o principal indicador de porte para as organizações. Neste estudo, esperava-se que as empresas com maior faturamento seriam aquelas mais organizadas e com menor risco de sobrevivência. Estas empresas são, em geral, mais organizadas e empregam mais. 
Tendência da produção (TP): variável qualitativa do tipo 1-cresceu ou 2-reduziu. Associa-se que empresas que possuam um crescimento na produção, neste caso, tenham oportunizado o aumento da demanda para o escoamento. Espera-se que esse crescimento esteja relacionado, principalmente, com aquelas empresas que tem o artesanato como fonte principal de renda e que estejam atendendo a clientela satisfatoriamente. Espera-se neste estudo que o menor risco de sobrevivência esteja positivamente correlacionado com o crescimento da produção.

Inovação dos produtos: (IP): a inovação é um indício de que a empresa está em processo de evolução e atualização. Empresas que não inovam tendem a fracassar ou sofrerem estagnação pela inovação dos concorrentes. Assim, as empresas que inovam tendem a reduzir seu risco de sobrevivência e, a inovação em um setor têxtil é de extrema importância, devido sua flexibilidade e tendências da moda.

\section{RESULTADOS}

A Tabela 1 informa como seriam classificados os indivíduos caso o modelo de risco de sobrevivência se deixasse guiar apenas pela situação em que se enquadra a maioria dos casos observados. Utilizando os procedimentos discorridos na metodologia, a amostra foi dividida em dois grupos: 42 no grupo de maior risco de sobrevivência e 27 no grupo com menor risco de sobrevivência. Isso significa que o modelo classificaria corretamente aqueles que atuam no comércio da tecelagem tradicional apenas para complementar a renda ou por gosto pela atividade, mas que não tem a atividade como fonte principal de trabalho e renda. A priori, espera-se que o grupo com maior risco de sobrevivência seja composto por MPE com menos de 5 empregados, com faturamento inferior a média mais um desvio padrão, entre outras características compostas nas variáveis utilizadas. Deixa-se claro que a classificação foi relativa entre as MPE da amostra. Nesse caso, o percentual geral de acerto nas classificações seria de aproximadamente $61 \%$. Esse quadro de classificação anterior à análise atua, portanto, como uma referência para avaliar a eficácia do modelo quando ele passa a operar com as variáveis independentes para predizer a que grupo pertence certo indivíduo.

Tabela 1 - Classificação inicial dos grupos antes da aplicação do modelo logit

\begin{tabular}{c|c|c|c|c}
\hline & \multicolumn{2}{c|}{ Risco de sobrevivência } & Percentagem Correta \\
\hline Grupos & Maior risco & Maior risco & Menor risco & \\
\hline & Menor risco & 42 & 0 & 100,0 \\
\hline & 27 & 0 & 0,0 \\
\hline
\end{tabular}

A Tabela 2 exibe o quadro da estatística Wald, que nesse primeiro momento está avaliando a significância da constante incluída no modelo. Do exposto, conclui-se que seria conveniente formular predições em função desse critério de classificação, visto que o nível de significância foi de 7,3\%. O objetivo dessa estatística é fornecer uma base de comparação que permita verificar se as variáveis independentes vêm melhorar a qualidade das predições. É importante considerar que sem incluí-las no modelo já se alcança um nível de acerto aproximado de $61 \%$ na classificação dos casos. Com elas, espera-se um percentual mais elevado. 
Tabela 2 - Estatística de Wald para a constante da função de regressão

\begin{tabular}{c|c|c|c}
\hline & B & Wald & Significância. \\
\hline Constante & $-0,442$ & 3,208 & 0,073 \\
\hline \multicolumn{2}{|c|}{ Fonte: Resultado da pesquisa }
\end{tabular}

É importante verificar se todas as variáveis aplicadas ao modelo podem explicar o fato de empresas terem maior ou menor probabilidade de sobrevivência. Caso se observe uma relação de significância entre o fenômeno e as variáveis incorporadas ao modelo, pode-se aproveitá-las na construção de um modelo voltado para identificar a probabilidade de um negócio ter mais chance de perpetuação no mercado. A manutenção das MPE da amostra mostra-se importante, segundo Santos e Silva (1997), devido à geração de renda e empregos dessa atividade. Sabe-se que isso refere-se a uma relação significativa, ou seja, algo que estatisticamente possa explicar a ocorrência do determinado evento. Por isso, nas próximas etapas serão exibidos os testes de significância, pois, com eles pode-se verificar se o modelo é capaz de realizar predições com a acurácia desejada.

Como evidencia a Tabela 3, uma das primeiras providências é testar a validade do modelo como um todo. Na regressão linear esse procedimento é realizado por meio da estatística F, cujo objetivo é testar a hipótese de que todos os coeficientes da equação são nulos. A confirmação dessa hipótese sugere que o modelo não serve para a estimação de valores para a variável dependente em função de valores conhecidos das independentes. Sim, porque nesse caso nenhum dos coeficientes seria significativamente diferente de zero.

Tabela 3 - Teste de Omnibus

\begin{tabular}{c|c|c|c}
\hline & Qui-quadrado & Graus de liberdade & Significância \\
\hline Step & 69,055 & 7 & 0,000 \\
\hline \multicolumn{3}{|c}{}
\end{tabular}

O modelo Qui-quadrado testa a hipótese de que todos os coeficientes da equação logística são nulos. O valor do modelo, como mostrado na Tabela 3 foi de 69,055. O Likelihood (-2LL) e a diferença entre os valores iniciais e finais desse indicador expressam a capacidade preditiva do modelo. Esse número corresponde à diferença entre o valor de (-2LL) obtido quando se inclui apenas a constante no modelo e o (-2LL) calculado após a inclusão de todas as variáveis independentes. Com a inclusão das variáveis independentes, espera-se que o Likelihood Value sofra uma redução estatisticamente significativa. Com 7 graus de liberdade verificou-se uma redução significativa e pode-se concluir que pelo menos um dos coeficientes da regressão logística é diferente de zero. Portanto, pode-se rejeitar a hipótese de que todos os parâmetros estimados são nulos. Em outras palavras, pode-se afirmar que eles contribuem para melhorar a qualidade das predições.

Na Tabela 4, os testes Cox \& Snell e Nagelkerke são considerados pseudos-R ${ }^{2}$. Eles procuram indicar a proporção das variações ocorridas no log da razão de chance que é explicada pelo conjunto das variáveis independentes. Assim o Cox \& Snell está indicando que cerca de $63,5 \%$ das variações ocorridas no $\log$ da razão de chance são explicadas pelo conjunto das variáveis independentes. Com significado semelhante ao coeficiente de determinação, o Nagelkerke considera que o modelo é capaz de explicar $85,7 \%$ das variações registradas na variável dependente.

Tabela 4 - Testes de Cox Snell e Nagelkerke

\begin{tabular}{c|c|c}
\hline-2 Log Likelihood & Cox \& Snell $R$ Square & Nagelkerke R Square \\
\hline $23,312(\mathrm{a})$ & 0,632 & 0,857 \\
\hline \multicolumn{2}{c}{ Fonte: Resultado da pesquisa }
\end{tabular}

Na Tabela 5, tem-se o indicador denominado teste Hosmer e Lemeshow. Trata-se de um teste qui-quadrado, cujo objetivo é testar a hipótese de que não há diferenças significativas entre 
os resultados preditos pelo modelo e os observados. Para tanto, dividem-se os casos em dez grupos, aproximadamente, iguais e comparam-se os valores observados com os esperados.

Seguindo uma distribuição qui-quadrado, o cálculo leva a uma estatística de 1,882 e um nível de significância de $98,4 \%$. Isso indica que os valores preditos não são significativamente diferentes dos observados. Como o teste pretende aceitar a hipótese de que não existem diferenças entre valores preditos e observados, têm-se mais um indício que o modelo pode ser utilizado para estimar a probabilidade de sobrevivência das MPE.

Tabela 5 - Teste de Hosmer and Lemeshow

\begin{tabular}{c|c|c}
\hline Qui-quadrado & Graus de liberdade & Significância \\
\hline 1,882 & 8 & 0,984 \\
\hline \multicolumn{2}{c|}{ Fonte: resultado da pesquisa }
\end{tabular}

Como se observa até aqui todos os testes foram favoráveis ao uso das variáveis independentes como estimadores do status de sobrevivência dos estabelecimentos comerciais da tecelagem tradicional. Sem incluir tais variáveis, o percentual de acertos seria de $61 \%$. Como a Tabela 6 mostra, incluindo as variáveis independentes o modelo possui um percentual de $94,2 \%$ de acerto com um nível de confiança de 5\%. Assim, estatisticamente é viável incluir as variáveis no modelo.

Tabela 6 - Classificação do modelo de regressão logística

\begin{tabular}{c|c|c|c|c}
\hline \multicolumn{2}{l|}{} & \multicolumn{2}{c|}{ Risco de sobrevivncia } & Porcentagem correta \\
\hline Grupos & Maior risco & Maior risco & Menor risco & \\
\hline & Menor risco & 20 & 2 & 95,2 \\
\hline \multicolumn{2}{|c|}{ Porcentagem total } & 2 & 25 & 92,6 \\
\hline
\end{tabular}

Como visto, todos os testes sugerem que, de forma geral, o modelo pode ser utilizado para estimar o risco de sobrevivência das MPE da tecelagem tradicional de Resende Costa. Resta ainda verificar a significância de cada coeficiente em particular. Afinal, é necessário verificar se cada um, realmente, pode ser utilizado como estimador de probabilidades. Para tanto, recorre-se novamente à estatística Wald. Como a variável independente tem apenas um grau de liberdade, para cada coeficiente procede-se ao seguinte cálculo:

$$
\text { Wald }=\left(\frac{b}{S . E}\right)^{2}
$$

onde,

b simboliza o coeficiente de uma variável incluída no modelo

S.E, o erro padrão a ele associado.

Como se observa na Tabela 7, o quadro seguinte sugere que nem todas as variáveis podem ser aproveitadas na composição do modelo, já que algumas não possuem o coeficiente diferente de zero. Os coeficientes das variáveis 'número de empregados', 'faturamento mensal', 'status da atividade' e a constante não foram diferentes de zero. Assim, a regressão será composta pelas variáveis 'tendência da produção', 'inovação dos produtos', 'tempo de atividade comercial' e 'curso e treinamento'. Em outras palavras, pode-se afirmar que cada um deles exerce efeito sobre a probabilidade de sobrevivência de um estabelecimento comercial pelo menos a um nível de significância de 5\%. 
Tabela 7 - Variáveis na equação

\begin{tabular}{|c|c|c|c|c|}
\hline & Coeficiente & Significância & \multicolumn{2}{|c|}{ Intervalo de confiança } \\
\hline Tendência da produção (TP) & $-18,84$ & 0,03 & 0,00 & 0 \\
\hline Inovação dos produtos (IP) & $-5,53$ & 0,01 & 0,00 & 0,20 \\
\hline Número de empregados (EP) & 1,35 & 0,13 & 0,64 & 23,23 \\
\hline Faturamento bruto mensal (FBM) & 0,00 & 0,58 & 1,00 & 1,00 \\
\hline Tempo de atividadecomercial(TAC) & 3,65 & 0,00 & 4,08 & 365,80 \\
\hline Status da renda (SR) & 18,36 & 1,00 & 0,00 & 0,00 \\
\hline Curso ou treinamento (CT) & $-4,29$ & 0,01 & 0,00 & 0,47 \\
\hline Constant & $-30,97$ & 1,00 & 0,00 & 0,00 \\
\hline
\end{tabular}

Fonte: Resultado da pesquisa

O Gráfico 2 mostra como foram classificados cada estabelecimento comercial da amostra na previsão. Percebe-se que devido ao elevado percentual de acerto do modelo, é quase imperceptível identificar quais estabelecimentos foram classificadas de maneira incorreta dentro do gráfico.

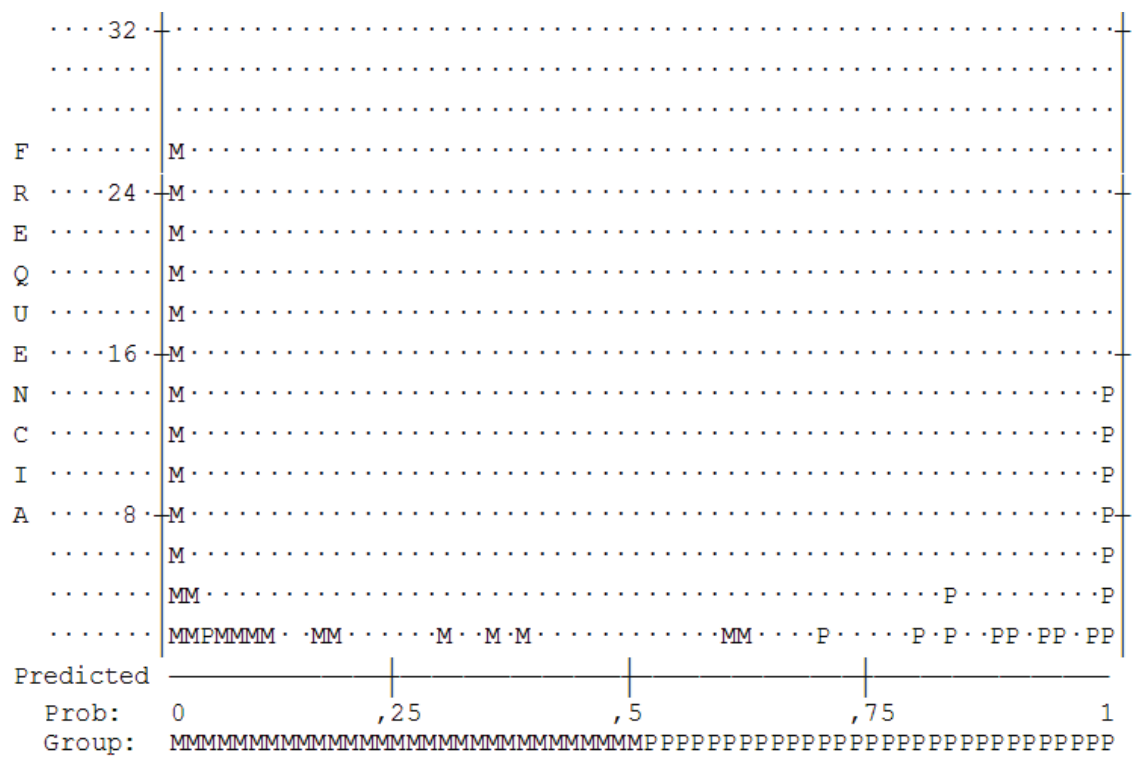

Símbolos: M - Maior risco de sobrevivência; P - Menor risco de sobrevivência (cada símbolo representa dois casos)

Gráfico 2 - Probabilidade dos casos

Submetido a todos os testes, verifica-se que o modelo está estatisticamente apto a ser utilizado na solução do problema de investigar a probabilidade de sobrevivência das MPE comerciais da tecelagem tradicional de Resende Costa. Desta forma, podem -se esboçar a equação da regressão logística com boa margem de segurança. Desta maneira pode-se excluir as variáveis número de empregados, faturamento mensal, status da renda e a constante. Fica a seguinte função de regressão representada pela equação 2:

$$
\begin{aligned}
& P(y)=\frac{1}{1+e^{-(-18,845 T P-5,535 I P+3,655 T A P-4,294 C T)}} \\
& P(y)=\frac{1}{1+e^{-(-18,845 T P-5,535 I P+3,655 T A P-4,294 C T)}} P(y)=\frac{1}{1+e^{-(-18,845 T P-5,585 I P+8,655 T A P-4,294 C T)}} \\
& P(y)=\frac{1}{1+\varepsilon^{-(-18,845 T P-5,585 I P+8,655 T A P-4,294 C T)}}
\end{aligned}
$$


Como se verifica na regressão linear, cada coeficiente deve ser interpretado como estimativa do efeito que uma variável independente produz sobre a dependente quando as demais se mantêm inalteradas. Entretanto, o modelo logístico é expresso em termos de logaritmos da razão de chance ou logit. Assim, cada coeficiente deve ser interpretado como o efeito que uma variação unitária sofrida pela variável independente tende a produzir sobre o logaritmo da razão de chance. O sinal do coeficiente é que vai determinar a direção da mudança, que pode ser aumentativa ou diminutiva. Já o efeito de cada coeficiente sobre a probabilidade é de natureza multiplicativa e vai depender do nível em que ela se encontrar.

Observa-se ainda, na Tabela 7, que os coeficientes negativos para as variáveis "tendência da produção" (TP) e "curso ou treinamento" (CT) mostram que uma variação positiva leva a uma redução do log de probabilidade. Soube-se na metodologia que para a variável TP, o valor 0 significa crescimento. Como o coeficiente foi negativo, o valor 1 (redução da produção) na equação para esta variável, mantendo as outras constantes, reduziria a probabilidade tendendo a função aproximar-se de um maior risco de sobrevivência $(Y=1)$. Para a variável "curso e treinamento", o raciocínio é análogo.

Pelo maior valor do coeficiente da variável tendência da produção (TP), pode-se perceber que é a que mais influencia na classificação das indústrias de Resende Costa. O valor 1 (redução da produção no último ano) na equação 2 , mantendo inalterada as outras variáveis, tende a provocar a maior redução da probabilidade de risco, o que significa o aumento do risco de sobrevivência. Assim, para um menor risco de sobrevivência espera-se, principalmente, que as MPE analisadas tenham auferido um crescimento da produção no último ano.

Como esperado, o tempo de atividade possui em coeficiente positivo, pois quanto mais tempo de atividade, mais a empresa será consolidada no mercado e mais experiente. $\mathrm{O}$ aumento do tempo de mercado aumenta a probabilidade, significando a redução do risco de sobrevivência.

Assim, as variáveis cujos coeficientes foram estatisticamente diferentes de zero, mostraram o comportamento esperado na análise preliminar, o que tende a sustentar os resultados alcançados.

\section{CONSIDERAÇÕES FINAIS}

Este estudo discorreu e apoiou a importância das MPE, bem como, mostrou a dinâmica da economia da atividade comercial da tecelagem tradicional de Resende Costa por meio dos fatores de risco de sobrevivência. Destacou-se que existe uma preocupação com os fatores relacionados à sobrevivência das MPE no Brasil, e especial neste município, devido à elevada mortalidade destas, principalmente, nos primeiros anos de funcionamento.

Por sua vez, utilizando de variáveis caracterizadoras das MPE, discorridas por estudiosos da área, foi possível implementar um modelo, estatisticamente significativo, para identificar o risco de sobrevivência destas empresas em Resende Costa (MG). Apesar da importância para caracterização de uma MPE, as variáveis que representam o número de empregados, o faturamento bruto mensal e importância da renda do comércio para a família (status da renda) não foram diferentes de zero, causando a exclusão destas do modelo de probabilidade. Para o modelo restaram as variáveis que representaram a tendência de crescimento da produção, a presença de inovação nos produtos, o tempo de atividade comercial e a participação em cursos ou treinamentos. 
A sobrevivência das MPE contribui para geração de emprego renda e redução da pobreza. Portanto, conhecer mais especificamente variáveis condicionantes da sobrevivência é algo essencial para a manutenção do nível de desenvolvimento do país de forma mais equitativa. É inédita, ao menos em papers brasileiros, a utilização da regressão logística para criação de um modelo de previsão de risco de sobrevivência em MPE. Assim, este estudo possui algumas limitações, entre as principais cita-se, primeiro, se um modelo determinístico poderia contribuir para identificação do risco de sobrevivência e, segundo, se as variáveis escolhidas na análise, seriam as mais adequadas para a composição do modelo.

Entre as principais limitações estão a analise de apenas uma cidade na amostra, o que limita que as conclusões possam ser realizadas para o estado ou país. Outra limitação está no fato de que não foi incluído nas entrevistas, questões sobre as características contábil-financeiras das empresas, fator considerados como mais importantes no estudo sobre fatores condicionantes da mortalidade das empresas brasileiras de acordo com SEBRAE (2007).

Sugere-se que este estudo seja utilizado como base para outros estudos que abordem mais variáveis, entre elas as financeiras. Como este estudo impossibilita generalizações por ser um caso de uma atividade de um mercado municipal, sugerem-se estudos mais aprofundados, com maior abrangência da área de estudo, de variáveis coletadas e outros setores de MPE. Destacase que por meio de outras aplicações, seja possível chegar a corroboração deste estudo, sua invalidação ou a reformulação de um melhor modelo preditivo, em decorrência da importância das MPE e da relevância dos modelos de previsão de risco.

\section{AGRADECIMENTOS}

Agradecemos ao Conselho Nacional de Desenvolvimento Científico e Tecnológico (CNPq) que proporcionou, por meio do financiamento do Edital 03/2008, as condições para a realização da pesquisa que deu origem a este trabalho

\section{REFERÊNCIAS}

ALBAGLI, S., BRITO, J. Glossário de Arranjos Produtivos locais. In: Projeto Arranjos Produtivos: Uma Nova Estratégia de Ação para o Sebrae. RedeSist/IE/UFRJ, 2002. 4-29 p.

AMORIM, M. Identificação de Arranjos Produtivos Locais no Ceará. In: (Orgs.) LASTRES, H. M. M.; CASSIOLATO, J. E.; MACIEL, M. L. Pequena Empresa: Cooperação e Desenvolvimento Local. Rio de Janeiro: Relume Dumará, 2003. p.17-59

BARBOSA, T. R. C. G. Introdução e características do processo de abertura de uma pequena empresa. Viçosa: Departamento de Administração da UFV, 2005. (Notas de aula).

BHOWMIK, S. Survey of Research on Street Vendors in Asia. Cambridge: Globalizing and Organizing, 2004. 76p

BROMLEY, R. Introduction the urban informal sector: why is it worth discussing? World Development, v.6,n.9, p. 1033-39, set. 1978.

CAMPOS, R. R.; CÁRIO, S. A. F.; NICOLAU, J. A.; VARGAS, G. T. Aprendizagem por Interação: Pequenas Empresas em Sistemas Produtivos e Inovativos locais. In: (Orgs.) LASTRES, H. M. M.; CASSIOLATO, J. E.; MACIEL, M. L. Pequena Empresa: Cooperação 
e Desenvolvimento Local. Rio de Janeiro: Relume Dumará, 2003. 345 p.

CHEN, M.A. Rethinking the Informal Economy: Linkages with the Formal Economy and the Formal Regulatory Environment. Cambridge: DESA Working paper. July, 2007.

CLEVERLY, W.O. Who is responsible for business failures? Healthcare financial management review, Westchester, Illinois, USA, , pp. 45-51, 2002.

CUSTÓDIO, A. V. Micro e Pequenas Empresas (MPE) Inseridas em Arranjo Produtivo Local - Um Estudo de Caso da Malacocultura no Estado de Santa Catarina. 2005. 168 f. Dissertação (Mestrado em Economia Industrial) - Universidade Federal de Santa Catarina, Florianópolis.

ERCOLIN, C.A. Fatores financeiros determinantes da mortalidade de micro e pequenas empresas. 2007. Dissertação (Mestrado em Administração) - Faculdade de Economia, Administração e Contabilidade, Universidade de São Paulo, São Paulo.

FILARDI, F. Estudos dos fatores contribuintes para a mortalidade precoce de micro e pequneas empresas na cidade de São Paulo. São Paulo, 2006. Tese (Doutorado em Administração) Programa de Pós-Graduação em Administração, Departamento de administração, faculdade de economia, administração e contabilidade da Universidade de São Paulo.

GUJARATI, D. N. Econometria Básica. São Paulo: Makron Books, 2000. 846 p.

LEMOS, C. Inovação para Arranjos e Sistemas Produtivas de MPME. In: VISLLASCHI, A. et al. Interagir para Competir: Promoção de Arranjos Produtivos e Inovativos no Brasil. Brasília: SEBRAE: FINEP/CNPq. 2002. 345 p.

LUNDVALL, B. A. et al. National systems of production, innovation and competence building. Summer Conference, Denmark, 2001. 45 p.

LUSSIER, R.N.; CORMAN, J. There are few differences between successful and failed small business. Journal of small business management, v.44, n.4, p.493-512, 2006.

MAGER, R.A. Avoiding the four deadly sins of business failure. Entrepreneurs Resource Guide, Advertising Supplement - San Diego Business Journal, n. 10, 2002.

MARTENS, C.D.P. A tecnologia de informação (TI) em pequenas empresas industriais do Vale do Taquari/RS. Porto Alegre. 2001. Dissertação (Mestrado em Administração) Universidade Federal do Rio Grande do Sul, Rio Grande do Sul.

MITULLAH, W. A review of street trade in Africa. Cambridge: Globalizing and Organizing (WIEGO), 2004.

PERRY, S. The relationship between written business plans and the failure of small business in the U.S. journal of Small Business Management, 39, v.3, p.201-208, 2001..

PREFEITURA MUNICIPAL DE RESENDE COSTA. Relatório de Contribuintes por Atividade Econômica - loja de artigos de artesanato. Resende Costa: Setor de Arrecadação, 2009. 3p. Relatório.

RIQUELME, H.; WATSON, J. Do venture capitalists'implicit theories on new business success/ failure have empirical validity? International Small Business Journal, v.20, n.4, pp. 395-420, 2002.

SANTOS, M. C. L. e SILVA, G. M. Tear: Artesanato de Resende Costa. São João del-Rei: Funrei, 1997. 
SANTOS, M. C. L., SILVA, G. M. e MORETTI, A. R. Artesanato: contando teares. São João del-Rei: Funrei, 1998.

SERVIÇO BRASILEIRO DE APOIO ÀS MICRO E PEQUENAS EMPRESAS -SEBRAE. Boletim estatístico de micro e pequenas empresas. Brasília: 2007. Disponível em: $<$ http://201.2.114.147/bds/BDS.nsf/03DE0485DB219CDE0325701B004CBD01/\$File/ NT000A8E66.pdf>. Acesso em: 20 fev. 2006.

SINDZINGRE, A. The Relevance of the Concepts of Formality and Informality: A Theoretical Appraisal. Oxford: EGDI, 2006. 234p.

SILVER, M. Estatística para administração. São Paulo: Atlas, 2000.

TOKMAN, V. E. An exploration into the nature of the informal-formal sector relationship. World Development, v. 6, n.10, p. 1065-75, 1978.

VIAPIANA, C. Fatores de sucesso e fracasso da micro e pequena empresa. Anais do II EGEPE, p.505-525, Londrina, Paraná, Nov. 2001. 


\section{ENDEREÇO DOS AUTORES:}

\section{Lucas Maia dos Santos}

Universidade Federal de Viçosa, Departamento de Administração.

Av. PH Rolfs Campus Universitário

Viçosa, MG - Brasil

36570-000

\section{Gustavo Melo Silva}

Universidade Federal de São João del Rei, Departamento de Ciências Administrativas e Contábeis. Av. Visconde do Rio Preto, s/n, Campus CTan Colônia do Bengo

São João Del Rei, MG - Brasil

$36300-000$

\section{Jorge Alexandre Barbosa Neves}

Universidade Federal de Minas Gerais, FAFICH, Departamento de Sociologia e Antropologia. Av. Antônio Carlos, 6.627, Campus Pampulha.

Belo Horizonte, MG - Brasil

31270-901 